\title{
A fluid definition of art
}

\section{Viewing images of liquid crystals as art raises complex questions.}

\section{Martin Kemp}

When does an image made by a researcher for scientific purposes become art - if at all? Over the ages, the beauty of scientific images has been widely recognized. Reading Robert Hooke's Micrographia (1665) gives us a sense of his aesthetic thrill faced with the wondrous images in the new device of the microscope. Like many of his contemporaries, Hooke would have recognized such miracles as the eye of the fly as the product of God's, or nature's, artistry. But does this mean that they are works of art in the normal sense of the term?

The question is implicitly raised by many of the striking products of modern scientific imaging techniques. And it is overtly posed by the claims of chemist John Goodby of the University of Hull, UK, that his microscopic images of liquid crystals (shown here) are "every bit as good as the kind of art you see in most galleries". Leaving aside the question of what is meant by "good" (and good for what?), his decision to start exhibiting his pictures as works of art plays into a complex series of shifting definitions of art in the modern era.

Until the twentieth century, the issue would not have arisen within the institutionalized definitions of art and science in post-Renaissance Western culture. But when artists decided to display everyday objects in art galleries - such as the signed urinal entitled Fountain by Marcel Duchamp in 1917 - the definition of art became very wobbly. The exhibiting of such 'ready-mades' and their enshrining in galleries and museums, in the collection of Duchamp's works in Philadelphia, for example, leaves us with a definition that extends little beyond the claim that anything is art that an artist claims is art - as is anything that viewers can look at as art.

Goodby's claims are of course more specific than merely saying that because he exhibits the liquid-crystal pictures as art, then they are art. $\mathrm{He}$

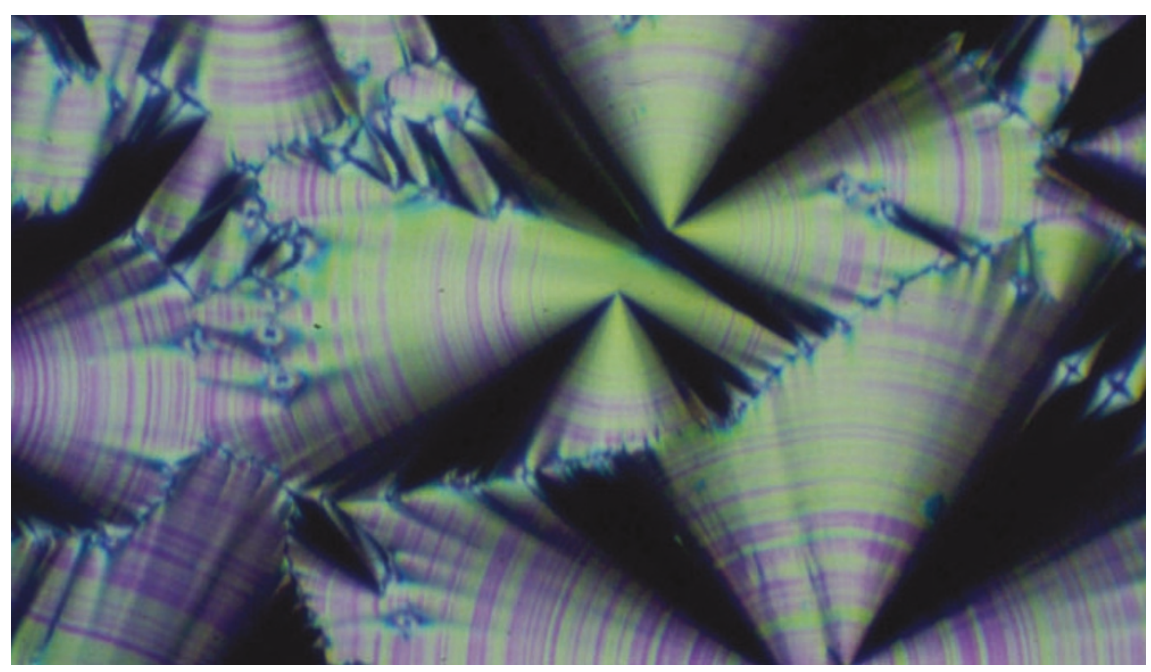

is implicitly setting his images in the context of modernist abstraction, in which paintings or sculptures are devoid of figurative subject matter and narratives. Indeed, the way that the great masters of abstraction have transformed what we are prepared to consider as art has radically enhanced our ability to appreciate the marvellous natural configurations revealed by modern scientific techniques.

The amount of artistic contrivance in Goodby's images far exceeds that in Duchamp's urinal. The selection of certain liquid crystals at certain stages in their intermediate state between solid and liquid, the setting up of the microscope to deliver certain visual qualities, and the choices involved in rendering and printing the pictures (regarding colours, textures, plasticity, scale and framing, for example) are all done to create the best effect. This is to say nothing of the way Goodby collages his images to produce images of birds and flowers.

I wonder how many scientists who use visual images prominently in publishing their work have not made some kind of aesthetic choice at some time or other. Certainly anything that features on the cover of Nature, in its current format, is designed to attract attention in ways that are comparable to the use of a painting on the cover of an art journal.

In the final analysis, should we worry about whether something is art or not? If it excites us, isn't that enough? My answer is drawn from a long historical perspective. The set definition of art as an aesthetic product devoid of practical function is actually comparatively recent (dating back to the late eighteenth century) and is limited to Western and Westernized societies. The art world has performed increasingly unconvincing conceptual gymnastics to accommodate everything that artists have recently thrown at it. If we stop being bothered by the question of whether something is art, and instead respond openly to the visual products that we are capable of making, we will be able both to agree with Goodby that his works are as 'good' as art, and say that any implicit competition between artists and scientist as makers of wonderful images is rather beside the point. Martin Kemp is professor of the history of art at the University of Oxford, Oxford OX1 1PT, UK, and co-director of Wallace Kemp Artakt. behind higher cognitive faculties will end in failure. Maybe there simply aren't any such mechanisms to be found. This is the view of 'functionalists' in cognitive science. They believe in large-scale patterns in human thinking of the kind portrayed in the familiar flowcharts, but they deny that there are any uniform physiological mechanisms to explain those regularities. Not that they assume any kind of spooky magic; rather, they argue that different mechanisms will underpin the regularities in different people.

From the functionalist point of view, asking about 'the physiological mechanism' responsible for scientific reasoning — to take a topic from the end of Changeux's book - is like asking for 'the low-level explanation' of why all word-processing programs work in roughly the same way. In truth, there isn't any one such explanation. Different programmers use different tricks, subject only to the constraint that their programs end up doing what word-processors have to do. Similarly, neural darwinism may ensure that our brains use different tricks to achieve roughly the same ends, subject only to the constraint that we all end up getting around in the world reasonably well.

Changeux has plenty to say about neural darwinism, and touches on functionalism in passing, but he doesn't quite spell out the connection between them. Still, his book presents a more satisfying picture of the brain than most of its competitors in this crowded market. On standard accounts, it can simply seem frustrating that we never get any bottom-up explanations of higher cognitive functions. If the structure of the brain is laid down by a definite genetic plan, then why can't we find out about the underlying mechanisms? Changeux's book fails to identify any such mechanisms too, but at least he gives us some insight into why the search for them may be doomed to permanent frustration.

David Papineau is professor of philosophy of science, King's College London, The Strand, London WC2R 2SL, UK. 